\title{
Parametric frequency mixing in a magnetoelastically driven linear ferromagnetic-resonance oscillator
}

\author{
C. L. Chang, ${ }^{1}$ A. M. Lomonosov, ${ }^{2}$ J. Janusonis, ${ }^{1}$ V. S. Vlasov, ${ }^{3,4}$ V. V. Temnov,${ }^{3,5,{ }^{*}}$ and R. I. Tobey ${ }^{1, \dagger}$ \\ ${ }^{1}$ Zernike Institute for Advanced Materials, University of Groningen, Groningen, The Netherlands \\ ${ }^{2}$ LAUM CNRS 6613, Université du Maine, 72085 Le Mans cedex, France \\ ${ }^{3}$ IMMM CNRS 6283, Université du Maine, 72085 Le Mans cedex, France \\ ${ }^{4}$ Syktyvkar State University named after Pitirim Sorokin, 167001, Syktyvkar, Russia \\ ${ }^{5}$ Fritz-Haber-Institut der Max-Planck-Gesellschaft, Abteilung Physikalische Chemie, Faradayweg 4-6, 14195 Berlin, Germany
}

(Received 10 October 2016; revised manuscript received 5 December 2016; published 15 February 2017)

\begin{abstract}
We demonstrate the linear frequency conversion of ferromagnetic resonance (FMR) frequency by optically excited elastic waves in a thin metallic film on dielectric substrates. Time-resolved probing of the magnetization directly witnesses magnetoelastically driven parametric second-harmonic generation, sum- and differencefrequency mixing from two distinct frequencies, as well as excitation of parametric resonances. Starting from the Landau-Lifshitz-Gilbert equations, we derive an analytical equation of an elastically driven (nonlinear) parametric oscillator and show that frequency mixing is dominated by the parametric modulation of the linear FMR oscillator.
\end{abstract}

DOI: 10.1103/PhysRevB.95.060409

Parametric behavior emerges in a wide range of periodically driven systems when their parameters are periodically modulated [1]. Examples can be found in nano-optomechanical [2-4] and microelectromechanical systems [5], (spin) wave dynamics [6], quantum circuitry [7], energy harvesting applications [8], and in line with our current report, magnetomechanical systems [9] including spin pumping capabilities [10]. The utility of parametric behavior has been shown for quantum limited detection, noise floor reduction, or low noise amplification of small signals [2,7].

Parametric phenomena in magnetization dynamics have also been extensively studied in the framework of spintronic and magnonic applications [11], where the downconversion of a microwave-driven uniform precession can generate two counterpropagating spin waves of varying frequency and wave vector. The onset of parametric behavior in these cases is monitored via the enhanced damping and linewidth changes of the ferromagnetic resonance (FMR) precessional motion. Furthermore, time domain probing of FMR precession modulated with multiple microwave electromagnetic fields leads to seeded parametric downconversion [12]. Additional studies along these lines have resulted in the generation and detection of a range of frequency mixing processes of both uniform precessional modes as well as higher energy spin waves [13-15], including frequency up- and downconversion.

Looking beyond microwave excitation, the overlapping frequency range of (surface) acoustic waves and magnetization precession provides for a unique opportunity to study their interactions and to explore physical processes where coherent elastic deformations could provide the necessary parametric modulation to drive complex magnetization dynamics. In recent years, magnetoelastic interactions have seen a resurgence of interest, and linear coupling between these degrees of freedom has been demonstrated [16-21] as well as one report of nonlinear effects [22].

\footnotetext{
*vasily.temnov@univ-lemans.fr

†r.i.tobey@rug.nl
}

An externally driven parametric oscillator is a classic example of a linear system with time-varying parameters, which can produce new frequencies without nonlinear interactions. In this Rapid Communication we present experimental evidence of the linear magnetoelastic frequency mixing between multiple coherent elastic deformations and the magnetization precession in a thin ferromagnetic film.

To explain our results, we perform analytical calculations of the Landau-Lifshitz-Gilbert equation, subject to the periodic excitation of a large amplitude, coherent elastic wave and show that the resulting dynamics can be described by an extended Mathieu equation for a nonlinear parametric oscillator. Simulation results based on our theory, where the nonlinear terms are neglected, show excellent correspondence with experimental results and allow us to identify a range of upconversion responses (enumerated below) as well as the downconverted precessional response commonly associated with parametric modulation.

The enabling feature of the present research is our recent demonstration of a simple optical technique that is able to generate multiple elastic waves utilizing the all-optical ultrafast transient grating (TG) technique [23], which facilitates the excitation and detection of multiple distinct elastic perturbations that propagate along the surface of a magnetic thin film. In our recent reports, we have identified them as the Rayleigh surface acoustic wave (SAW) and the surface skimming longitudinal wave (SSLW), oscillating at distinct frequencies $\omega_{S A W}$ and $\omega_{S S L W}$. These acoustic transients interact simultaneously with the FMR precession in a nickel film. Varying the applied magnetic field and underlying substrate material allows us to engineer the frequencies and relative elastic excitation strength of the two waves, to experimentally observe sum and difference frequency generation (SHG and DFG at $\omega_{ \pm}=\omega_{S S L W} \pm \omega_{S A W}$, respectively) and secondharmonic generation $\left(\mathrm{SHG}\right.$, both for $\omega_{S H G}=\omega_{S A W}+\omega_{S A W}$ and $\left.\omega_{S H G}=\omega_{S S L W}+\omega_{S S L W}\right)$.

In the TG geometry the sample is excited by two, spatially and temporally coincident optical pulses generating a spatially periodic, instantaneous excitation. Due to thermoelastic 

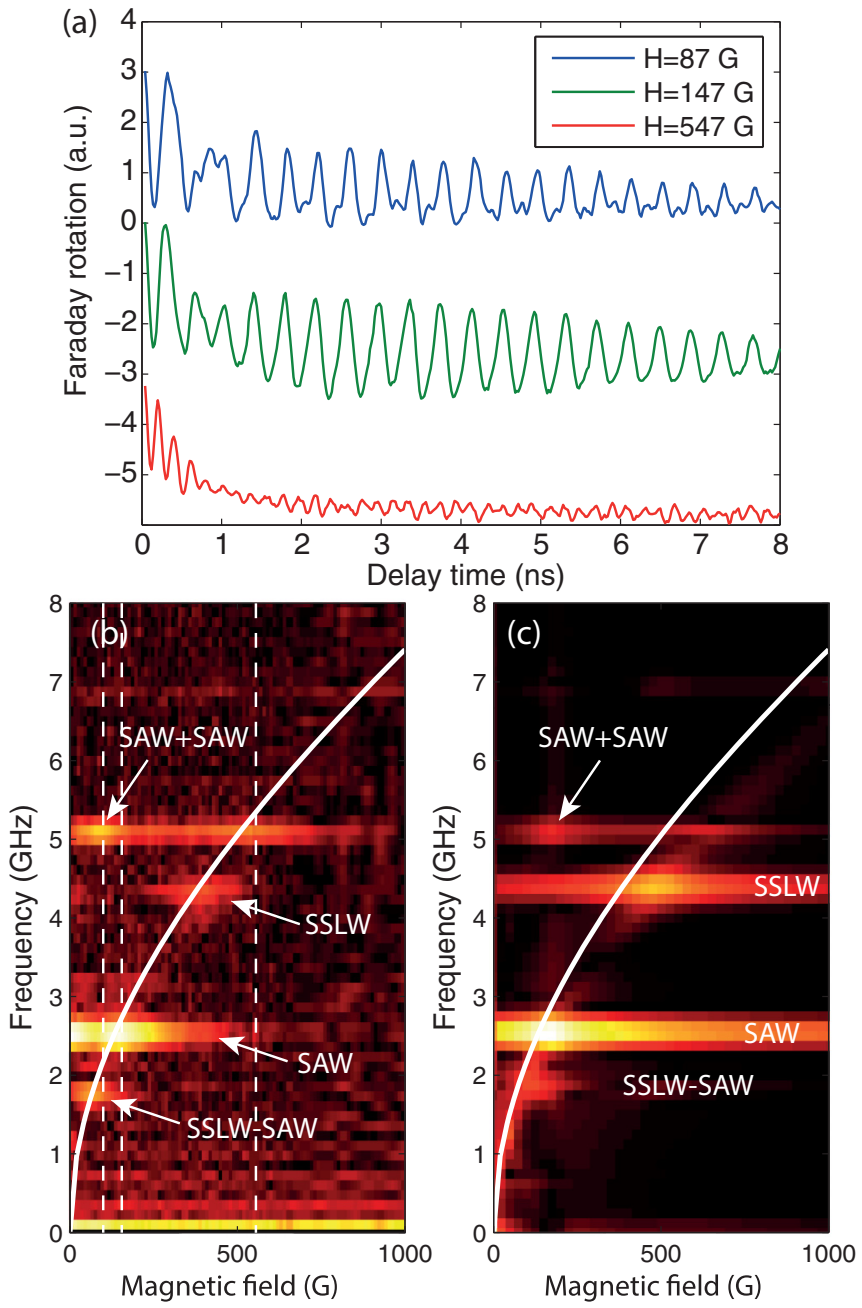

FIG. 1. (a) The time-resolved Faraday rotation for three representative applied fields, exhibiting a range of magnetoelastic responses. (b) The Fourier spectra of Faraday time traces vary strongly with the applied magnetic field, and show maxima when resonant with the elastic driving fields (linear response) as well as at their sum and difference frequencies. Magnetoelastic frequency mixing is observed at the second-harmonic frequency of the Rayleigh surface acoustic wave (SAW) and difference frequency with a weaker surface skimming longitudinal wave (SSLW) wave. (c) The numerical solution of the parametric oscillator equation [Eq. (1)] evidences the same behavior. The white solid line marks the FMR frequency $\Omega_{0}(H) / 2 \pi$, while vertical dashed lines identify the time traces in (a).

mechanisms, large amplitude elastic waves propagate along the surface of the film (as well as into the bulk of the film). A time-delayed probe measures the magnetization of the sample based on the rotation of the polarization of the transmitted beam (Faraday configuration), and is sensitive to the out-of-plane component of the magnetization as it precesses under the action of the elastic waves.

As an initial demonstration of magnetoelastic nonlinearities, we revisit the $\mathrm{Ni} / \mathrm{MgO}$ sample configuration we first described in Janušonis et al. [24]. On this substrate, the TG excitation leads to a strong Rayleigh SAW and a barely visible SSLW (see, e.g., [25]). Figure 1(a) shows the dependence of the magnetization precession amplitude (Faraday rotation, vertically offset) on the magnitude $H$ of the in-plane applied external magnetic field tilted by angle $\phi=30^{\circ}$ with respect to the TG wave vector. The magnetic field allows for tuning the thin-film FMR frequency following the Kittel formula $\Omega_{0}=$ $\gamma \mu_{0} \sqrt{H\left(H+M_{0}\right)}$, where $M_{0}$ is the saturation magnetization in nickel and $\gamma$ is the gyromagnetic ratio. For ease of visualization, the spectral amplitude of Fourier transforms of individual scans taken over the entire range of magnetic field are displayed in a two-dimensional map as shown in Fig. 1(b). Within the range of magnetic field up to $1000 \mathrm{G}$, the FMR frequency can be tuned to the underlying elastic frequencies (2.55 GHz for the SAW and weakly at $4.35 \mathrm{GHz}$ for the SSLW), their difference $(1.8 \mathrm{GHz})$ and sum $(6.9 \mathrm{GHz})$ frequencies, as well as the SAW second-harmonic frequency $(5.10 \mathrm{GHz})$. The elastic frequencies are determined by the underlying substrate material and are fixed once a TG period $\Lambda$ is experimentally selected and shown here for the case of $\Lambda=2.2 \mu \mathrm{m}$. For this combination of metal and substrate, the elastic amplitude of the SAW is far larger than that of the SSLW, resulting in a large precession signal for the linear response at $2.55 \mathrm{GHz}$ and its second harmonic $(5.10 \mathrm{GHz})$, and far weaker sum and difference frequency mixing signals with the weak SSLW. We therefore consider this as a monochromatic elastic wave (SAW) with a small additional contribution of the SSLW.

Theoretical analysis of elastically driven Landau-LifshitzGilbert equations [27] shows that a relatively moderate value of magnetostriction coefficient $b_{1}=1.5 \times 10^{5} \mathrm{~J} / \mathrm{m}^{3}$ in nickel results in a small-angle FMR precession around the external magnetic field (applied in the $x y$ plane). The linearization of Landau-Lifshitz-Gilbert equations in the vicinity of the equilibrium magnetization direction (see the Supplemental Material [26] for derivation) leads to an equation of a driven parametric oscillator,

$$
\frac{d^{2} m}{d t^{2}}+\Gamma_{0} \frac{d m}{d t}+\left[\Omega_{0}^{2}+\Omega_{1}^{2} e_{x x}(t)\right] m=F_{0} e_{x x}(t)
$$

for the in-plane component $m=M_{y}(t) / M_{0}$ of the timedependent magnetization vector $\vec{M}(t)$. The dominant term $e_{x x}(t)$ of the elastic strain represents a sum of two contributions: a large amplitude, time periodic, SAW excitation at frequency $\omega_{S A W}$ and a rapidly decaying SSLW excitation in line with our previous Green's function calculation of the elastic response [25].

Equation (1) represents an approximation of a more complicated equation [Eq. (4) in the Supplemental Material]. A detailed analysis shows that the damping term $\Gamma(t)=$ $\Gamma_{0}+\Gamma_{1} e_{x x}(t)$ is modulated by the elastic strain $e_{x x}(t)$ as well and that there exist high-order nonlinear terms proportional to $m^{2} e_{x x}$ and $m \frac{d m}{d t} e_{x x}$. However, the dominant terms in the sense of frequency mixing are the parametric modulation of FMR frequency $\Omega^{2}(t)=\Omega_{0}^{2}+\Omega_{1}^{2} e_{x x}(t)$ and the external driving force $F_{0} e_{x x}(t)$. An intrinsic property of our methodology is that the parametric modulation $\Omega_{1}^{2}(H, \phi) e_{x x}(t)=$ $\frac{\gamma^{2} \mu_{0} b_{1}}{M_{0}}\left(H+M_{0}-\left[3 H+2 M_{0}\right] \cos ^{2} \phi\right) e_{x x}(t)$ and the external driving force $F_{0}(H, \phi) e_{x x}(t)=\frac{\gamma^{2} \mu_{0} b_{2}}{2 M_{0}}\left(H+M_{0}\right) \sin 2 \phi e_{x x}(t)$ are both proportional to the strain amplitude $e_{x x}(t)$. However, their ratio $F_{0} / \Omega_{1}^{2} \propto\left(H+M_{0}\right) \sin 2 \phi /\left[H+M_{0}-\right.$ $\left.\left(3 H+2 M_{0}\right) \cos ^{2} \phi\right]$, which determines the relative strength of parametric modulation to driving force, does not depend 

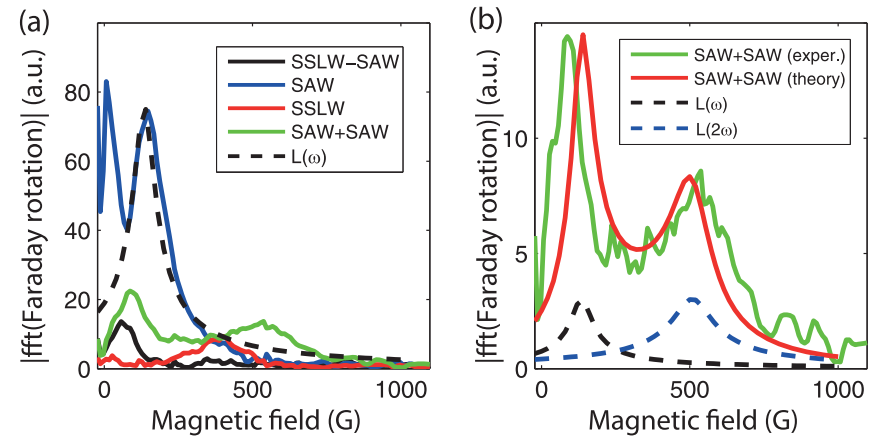

FIG. 2. (a) Horizontal cross sections of experimental [Fig. 1(b)] plots corresponding to SAW and SSLW frequencies as well as their difference (SSLW - SAW) and SAW second-harmonic $(\mathrm{SAW}+\mathrm{SAW})$ frequencies. The dashed line shows the SAW Lorentzian $L(\omega)$. (b) The strongest nonlinear mixing signal (SAW+SAW) is well approximated by an analytical approximation [Eq. (2)]; it displays two maxima corresponding to the resonances $\Omega_{0}(H=150 \mathrm{G})=\omega$ and $\Omega_{0}(H=520 \mathrm{G})=2 \omega$.

on strain and can be adjusted by either changing the magnitude of the applied field, $H$, and/or the orientation $\phi$ of the external magnetic field with respect to the TG wave vector.

In Fig. 1(c), we show the results of the numerical solution of Eq. (1). The solution of the equation results in a time-, angle-, and field-dependent time trace, which is subsequently Fourier transformed and displayed as a spectral amplitude. In comparison to Fig. 1(b), we note the exceptional similarity between the experimental data and the calculated response, and in particular the appearance of harmonics of the underlying elastic waves. In the simulation, the SAW and SSLW frequencies are a posteriori extracted from the experimental data and the SSLW amplitude and decay time are calculated by the Green's function formalism [23]. The parametrically driven upconversion and downconversion are the results of the simulation.

Figure 2(a) shows the dependence of the Fourier spectra in Fig. 1(b) on the magnetic field at selected frequencies corresponding to the acoustic SAW, and SSLW, as well as the parametrically driven responses of SSLW - SAW and SAW +SAW frequencies. The strongest SAW signal displays a resonance at $H=250 \mathrm{G}$. As discussed previously for this material heterostructure, the largest signal corresponds to the SAW excitation which is well approximated by a Lorentzian line shape (dotted line in the figure). In Fig. 2(b) we take a closer look at the dependence of the SHG signal $(\mathrm{SAW}+\mathrm{SAW})$ at $5.10 \mathrm{GHz}$ as a function of the magnetic field, which displays two pronounced maxima corresponding to the $\Omega_{0}=\omega$ and $\Omega_{0}=2 \omega$, both in the experiment and in the numerical simulation. Here $\omega$ denotes the frequency of the surface acoustic wave. In order to understand the physical origin of this dependence we have applied first-order perturbation theory to Eq. (1) assuming $e_{x x}(t)$ to be a small parameter. Assuming a monochromatic elastic driving force (SAW only) $e_{x x}(t)=e_{x x, 0} \exp (i \omega t)$ we obtained the following analytical expression for the first-order perturbation correction
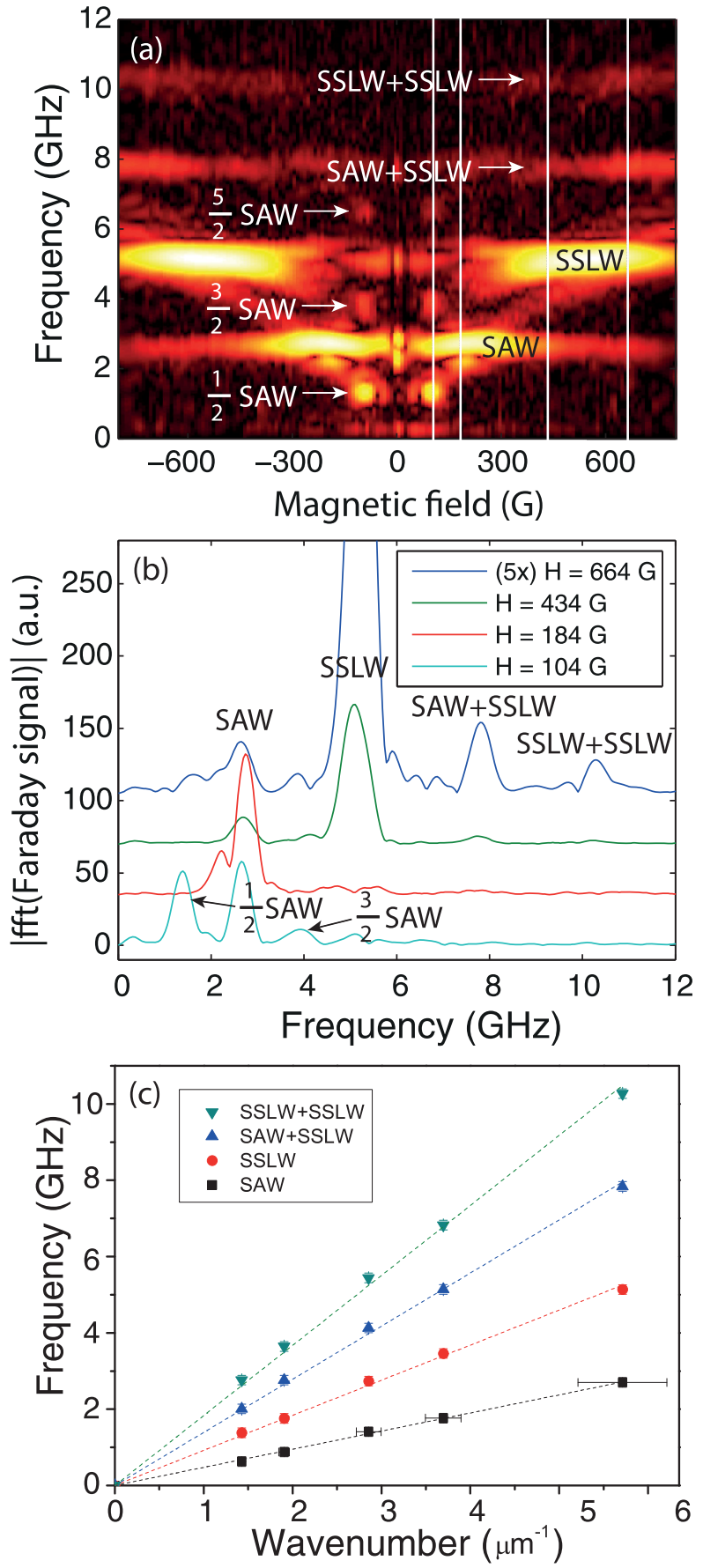

FIG. 3. (a) On glass substrates, two strong elastic waves are generated (SAW and SSLW) allowing the observation of sum frequencies at SAW+SSLW $(7.8 \mathrm{GHz})$ and SSLW+SSLW $(10.2 \mathrm{GHz})$ in addition to the linearly activated precession at 2.7 and $5.1 \mathrm{GHz}$. Additionally, conventional parametric resonances at $1 / 2,3 / 2$, and $5 / 2$ of SAW frequency are witnessed. (b) The attribution of these frequencies is unambiguously verified by the analysis of vertical cross sections for several values of magnetic field, providing a $\pm 0.1 \mathrm{GHz}$ error bar for all frequencies. (c) Dispersion relations for several grating periodicities. The resultant slope indicates the velocity of the excitation, which for the two lower modes, correspond to the propagation velocity of the underlying elastic waves. For the sum frequencies, the extracted "velocities" indicate that higher precessional frequencies always occur at the sum of the lower elastic responses. 
oscillating at frequency $2 \omega$ :

$$
m(2 \omega) \propto \frac{1}{\Omega_{0}^{2}-\omega^{2}+i \omega \Gamma_{0}} \frac{\Omega_{1}^{2}}{\Omega_{0}^{2}-4 \omega^{2}+2 i \omega \Gamma_{0}},
$$

which represents a product of two Lorentzians $L(\omega)$ and $L(2 \omega)$. Therefore, in analogy to nonlinear optics [28] the spectral dependence of the second-order susceptibility displays two resonances at frequencies $\omega$ and $2 \omega$, respectively. However, whereas in nonlinear optics SHG is caused by $\chi^{(2)}$ nonlinearities in the wave equation, in magnetoelastics it originates from the joint action of the parametric modulation of the FMR frequency and periodic external driving, both at the same frequency $\omega$.

The above analytical treatment neglects the second driving frequency at the SSLW, which is necessary to explain the sum and difference frequency wave mixing. In contrast to the longlived SAW, the amplitude of the SSLW rapidly decays on a time scale of about 1-3 ns, depending on the elastic constants of the substrate, TG wavelength, and nickel thickness. However, replacing the first Lorentzian $L(\omega)$ in Eq. (2) by a sum of two Lorentzians, $L(\omega)+L\left(\omega_{S S L W}\right)$, allows one to approximate the experimental data for SSLW - SAW in Fig. 2(a) as well.

Engineering the elastic properties of the dielectric substrate can be used to enhance existing, or create additional parametric responses. In contrast to the $\mathrm{MgO}$ substrate, where the SAW excitation dominates, a similar nickel/glass structure displays markedly different behavior due to the strong contribution of the SSLW excitation [25], providing additional opportunities to tune the nonlinear responsivity. As a demonstration, we perform the same measurements on the Ni/glass structure with a $1.1 \mu \mathrm{m}$ period, which results in a more efficient magnetoelastic frequency mixing as shown in Fig. 3(a). In addition to the linear SAW and SSLW excitations, there now exists two responses at 7.8 and $10.2 \mathrm{GHz}$ which we recognize as the precession response due to the sum frequencies of the underlying elastic waves, SAW+SSLW and SSLW+SSLW (due to the particularities of the elastic properties of the glass, SAW+SAW excitation overlaps nearly perfectly with the linear SSLW response and is thus not evident). In addition to these sum frequencies, the response on glass is marked by the appearance of precessional amplitude at parametric resonances occurring at $1 / 2,3 / 2$, and $5 / 2$ of the SAW frequency as indicated in the figure. The data in Fig. 3 illustrate the effect for a magnetic field angle of $\phi=7.5^{\circ}$ (magnetic field nearly collinear to the TG wave vector), where parametric driving dominates. Likewise, turning the magnetic field angle to larger values suppresses the parametric response as shown in the Supplemental Material for $\phi=60^{\circ}$.
Finally, we aggregate the results of several grating periodicities $(1.1,1.7,2.2,3.3$, and $4.4 \mu \mathrm{m})$ on the glass substrate, all of which show sum-frequency generation, to show the scaling of precessional frequency as a function of grating wave number. The extracted velocities for the two lower branches are $3000 \pm 125$ and $5900 \pm 110 \mathrm{~m} / \mathrm{s}$ in line with our previous measurement and published data for the Rayleigh SAW and longitudinal velocity in glass. For the two upper branches, we extract values of $8900 \pm 230$ and $11800 \pm 300 \mathrm{~m} / \mathrm{s}$ which overlap, within errors, with the sum frequencies SAW+SSLW and SSLW+SSLW. Velocity values are obtained by zero-intercept linear fits of the dispersion curves, while the horizontal and vertical error bars on the data account for the uncertainty in excitation grating period and frequency uncertainty due to the finite measurement time, respectively. The dispersion relations should be understood in the framework of elastic propagation and the resonant precession that they drive. While the two lower branches represent both elastic velocity and precessional frequency, the upper branches should only be associated with the precession of magnetization at the sum frequencies of the underlying elastic waves.

In summary, we have demonstrated the general feature of parametric excitation of magnetization precession under the action of a driving elastic field. Under an applied field, the particularities of the response can be tuned to accentuate or amplify either the sum, difference, or parametric resonance frequencies. The range of responses can be further selected by a careful choice of substrate material, which selects the relative strengths of the active elastic waves. To corroborate our experimental results, we calculate the response of magnetization under the action of elastic waves to arrive at the equation for a parametric oscillator. Numerical and analytical calculations find excellent qualitative agreement to the data. This initial demonstration opens the door to more complex elastic wave [29] control over magnetization that could herald the emergence of extremely broadband, widely tunable, control of magnetization precession, including magnetization reorientation, and elastic activation of quantized magnonic modes. Furthermore, the presented methodology can be used to investigate novel materials with unknown magnetoelastic properties. Thin films of magnetic MAX phases with negligible magnetocrystalline anisotropy and possibly high magnetostrictive coupling could be good candidates for future research [30].

Funding from Stratégie Internationale "NNN-Telecom" de la Région Pays de La Loire, ANR-DFG "PPMI-NANO" (ANR-15-CE24-0032 and DFG SE2443/2) and Alexander von Humboldt Stiftung is highly appreciated.
[1] E. Butikov, Eur. J. Phys. 25, 535 (2004).

[2] D. Rugar and P. Grutter, Phys. Rev. Lett. 67, 699 (1991).

[3] M. Aspelmeyer, T. J. Kippenberg, and F. Marquard, Rev. Mod. Phys. 86, 1391 (2014).

[4] L. Papariello, O. Zilberberg, A. Eichler, and R. Chitra, Phys. Rev. E 94, 022201 (2016).
[5] W. Zhang, R. Baskaran, and K. L. Turner, Sens. Actuators A 102, 139 (2002).

[6] V. S. L'vov, Wave Turbulence Under Parametric Excitation, Springer Series in Nonlinear Dynamics (Springer, Berlin, Heidelberg, 1994).

[7] M. A. Castellanos-Beltran, K. D. Irwin, G. C. Hilton, L. R. Vale, and K. W. Lehnert, Nat. Phys. 4, 929 (2008). 
[8] Y. Jia, S. Du, and A. A. Seshia, Sci. Rep. 6, 30167 (2016).

[9] X. Zhang, C.-L. Zou, L. Jiang, and H. X. Tang, Sci. Adv. 2, 1501286 (2016).

[10] H. Keshtgar, M. Zareyan, and G. Bauer, Solid State Commun. 198, 30 (2014).

[11] A. A. Serga, A. V. Chumak, and B. Hillebrands, J. Phys. D: Appl. Phys. 43, 264002 (2010).

[12] A. Y. Elezzabi and S. E. Irvine, Appl. Phys. Lett. 82, 2464 (2003).

[13] A. Capua, C. Rettner, and S. S. P. Parkin, Phys. Rev. Lett. 116, 047204 (2016).

[14] T. Gerrits, P. Krivosik, M. L. Schneider, C. E. Patton, and T. J. Silva, Phys. Rev. Lett. 98, 207602 (2007).

[15] H. Bauer, P. Majchrak, T. Kachel, C. Back, and G. Woltersdorf, Nat. Commun. 6, 8274 (2015).

[16] M. Weiler, L. Dreher, C. Heeg, H. Huebl, R. Gross, M. S. Brandt, and S. T. B. Goennenwein, Phys. Rev. Lett. 106, 117601 (2011).

[17] L. Thevenard, C. Gourdon, J. Y. Prieur, H. J. von Bardeleben, S. Vincent, L. Becerra, L. Largeau, and J.-Y. Duquesne, Phys. Rev. B 90, 094401 (2014).

[18] L. Dreher, M. Weiler, M. Pernpeintner, H. Huebl, R. Gross, M. S. Brandt, and S. T. B. Goennenwein, Phys. Rev. B 86, 134415 (2012).

[19] J.-W. Kim, M. Vomir, and J.-Y. Bigot, Phys. Rev. Lett. 109, 166601 (2012).

[20] A. V. Scherbakov, A. S. Salasyuk, A. V. Akimov, X. Liu, M. Bombeck, C. Brüggemann, D. R. Yakovlev, V. F. Sapega, J. K. Furdyna, and M. Bayer, Phys. Rev. Lett. 105, 117204 (2010).
[21] J. V. Jäger, A. V. Scherbakov, T. L. Linnik, D. R. Yakovlev, M. Wang, P. Wadley, V. Holy, S. A. Cavill, A. V. Akimov, A. W. Rushforth et al., Appl. Phys. Lett. 103, 032409 (2013).

[22] D. Afanasiev, I. Razdolski, K. M. Skibinsky, D. Bolotin, S. V. Yagupov, M. B. Strugatsky, A. Kirilyuk, T. Rasing, and A. V. Kimel, Phys. Rev. Lett. 112, 147403 (2014).

[23] J. Janušonis, T. Jansma, C. L. Chang, Q. Liu, A. Gatilova, A. M. Lomonosov, V. Shalagatskyi, T. Pezeril, V. V. Temnov, and R. I. Tobey, Sci. Rep. 6, 29143 (2016).

[24] J. Janušonis, C. L. Chang, P. H. M. van Loosdrecht, and R. I. Tobey, Appl. Phys. Lett. 106, 181601 (2015).

[25] J. Janušonis, C. L. Chang, T. Jansma, A. Gatilova, V. S. Vlasov, A. M. Lomonosov, V. V. Temnov, and R. I. Tobey, Phys. Rev. B 94, 024415 (2016).

[26] See Supplemental Material at http://link.aps.org/supplemental/ 10.1103/PhysRevB.95.060409 for a derivation of the nonlinear parametric oscillator equation from LLG equations and a comparative analysis of nonlinear and parametric contributions therein.

[27] O. Kovalenko, T. Pezeril, and V. V. Temnov, Phys. Rev. Lett. 110, 266602 (2013).

[28] V. V. Temnov, I. Razdolski, T. Pezeril, D. Makarov, D. Seletskiy, A. Melnikov, and K. A. Nelson, J. Opt. 18, 093002 (2016).

[29] F. J. R. Schulein, E. Zallo, P. Atkinson, O. G. Schmidt, R. Trotta, A. Rastelli, A. Wixforth, and H. J. Krenner, Nat. Nanotechnol. 10, 512 (2015).

[30] R. Salikhov, A. Semisalova, A. Petruhins, A. Ingason, J. Rosen, U. Wiedwald, and M. Farle, Mater. Res. Lett. 3, 156 (2015). 